\title{
Growth parameters affect geochemical proxies in massive corals: Impacts on climate reconstructions
}

\author{
MARINE CANESI ${ }^{1,2}$, ERIC DOUVILLE ${ }^{3}$, LOUISE \\ BORDIER $^{3}$, ARNAUD DAPOIGNY ${ }^{3}$, GNINWOYO \\ COULIBALY $^{1}$, ANNE-CATHERINE SIMON ${ }^{4}$, MATHIEU \\ AGELOU $^{4}$, PAOLO MONTAGNA ${ }^{5}$, DENIS ALLEMAND ${ }^{2}$ \\ AND STÉPHANIE REYNAUD ${ }^{2}$ \\ ${ }^{1}$ Laboratoire des Sciences du Climat et de l'Environnement \\ (CEA-CNRS-UVSQ) \\ ${ }^{2}$ Centre Scientifique de Monaco \\ ${ }^{3}$ LSCE/IPSL, CEA-CNRS-UVSQ \\ ${ }^{4}$ Université Paris-Saclay (CEA) \\ ${ }^{5}$ Institute of Polar Sciences (ISP-CNR) \\ Presenting Author: marine.canesi@1sce.ipsl.fr
}

Calcifying organisms such as massive scleractinian corals precipitate aragonite to build their hard skeleton. During skeletal growth, these organisms record environmental parameters (e.g. seawater temperature and $\mathrm{pH}$ ) that are imprinted in the biogenic aragonite. Therefore, corals can be used as natural archives to reconstruct past environmental changes. In particular, elemental ratios (e.g. $\mathrm{Sr} / \mathrm{Ca}, \mathrm{Li} / \mathrm{Mg}, \mathrm{U} / \mathrm{Ca}, \mathrm{B} / \mathrm{Ca}$ ) and boron isotopes in massive tropical coral colonies provide long term records of past ocean temperature and carbonate chemistry properties, and also a way to evaluate the impacts of global changes on coral growth. However, the biomineralization process exerts a physiological control over the geochemistry of coral skeleton, termed "vital effects", which may bias reconstructions. Although the use of multi-proxy (e.g. $\mathrm{Sr} / \mathrm{Ca}-\mathrm{Li} / \mathrm{Mg}$ ) or $\mathrm{Sr}-\mathrm{U}$ methods has improved the reliability and accuracy of coral-based seawater temperature reconstructions by reducing the physiological effects, the coral biology still affects part of the geochemical signal and hence paleo-reconstructions.

That is why, it is crucial to consider also the evolution of the growth parameters (e.g. density, linear extension and calcification rates) to correctly interpret the coral geochemistry.

The aims of this study are to (1) quantify the growth parameters of the massive Porites and Diploastrea corals, (2) evaluate the impacts of "vital effects" on the skeletal geochemistry and (3) combine different approaches to refine the use of coral proxies.

For this, we investigated the elemental and isotopic compositions and the growth parameters of several specimens of massive corals from the Pacific Ocean and cultured in controlled conditions. Preliminary results on a Porites colony grown first in-situ and then in aquaria show strong differences in density and linear extension rate associated to abrupt changes in elemental and isotopic composition. Based on these results, we decided to evaluate the impact of growth parameters over long-term (decadal time scale) on climate reconstructions based on Porites and Diploastrea colonies from Palau, Taiwan and Papua New Guinea. 